\title{
On the performance modeling of outage probability in CDMA wireless networks
}

\author{
M. E. Ekpenyong ${ }^{1, *}$, G. Eromosele ${ }^{1}$ and J. Isabona ${ }^{2}$ \\ ${ }^{1}$ Dep. of Computer Scienc, University of Uyo, PMB. 1017 520003, Uyo, Nigeria \\ ${ }^{2}$ Dep. of Basic Science, Benson Idahosa University, PMB 1100, Benin City, Nigeria
}

Received 24 September 2010; Accepted 27 January 2011

\begin{abstract}
Outage probability is a key performance measure for real time traffic in wireless networks and is often considered as more critical than drop call probability. In this paper, we study the outage probability in a wireless mobile environment, where the interferers are distributed on the ground. Taking into consideration the background noise and the cell radius, we formulate an outage probability model coupled with observed data from Airtel Nigeria formally Zain Communications Limited, a Telecommunication company operating in Nigeria and simulate the system's performance using the Java programming language. We also present a geographical information system (GIS) solution showing the distribution of signal outages at various base stations in Akwa Ibom State (East region) of Nigeria for observed and simulated data. A performance evaluation of these data reveals that our model could greatly improve the system's performance. The results obtained allow us to study the stability of the network and evaluate the network in a more efficient and accurate manner.
\end{abstract}

Keywords: Co-channel interference, signal outage, spatial traffic, spectral efficiency.

\section{Introduction}

Wireless communication networks have recently undergone extensive studies both from the theoretical and communication perspectives, including the development of practical transmission strategies and fundamental boundaries (capacity) in order to optimize the network [1]. The performance of wireless networks is greatly impaired by cochannel interference among several links (several mobile users (MUs) operating simultaneously in a wireless communication network). This effect of interference at the physical layer has also been extensively covered in $[2,3,4,5,6,7]$.

CDMA is a digital technology that uses spread spectrum techniques. It does not assign specific frequencies to users; rather, every channel uses the full available spectrum. However, the frequency spectrum allocated for cellular communication is limited and as such, the success of today's cellular networks is mainly due to frequency reuse (because callers make use of the same frequency spectrum in an overlapping time). This causes an increase in intra-cell (users within a cell) as well as inter-cell (users in adjacent cells) interference. As the number of active callers (or mobile stations) increase in a cell (or base station), at a certain level, the signal-to-interference ratio (SIR) at the receiver gets below a stipulated minimum [8] and will require each user to maintain a high level of SIR for satisfactory reception, otherwise the user experiences an outage. But for a given maximum allowable transmission power from the base station, an increase in the required power (signal reception) results in a decrease in the maximum distance a MS can be from the BS. This implies that only mobile stations (MSs)

\footnotetext{
*E-mail address: ekpenyong_moses@yahoo.com

ISSN: 1791-2377 @ 2011 Kavala Institute of Technology. All rights reserved.
}

closer to the base station (BS) will acquire the required power (i.e. higher power) to receive signal and communicate. This eventually leads to decrease in network capacity as the coverage deceases and results in a reduction in battery life, because of additional effort the MS adds to meet up communication by increasing its power. Based on this, there is need for power control algorithms to allocate equal power to all MS and hence widen the coverage of the cell, thereby increasing the capacity of the network.

This paper therefore seeks to address the following questions:

(i) How can co-channel interference be controlled to minimize outages?

(ii) What strategies exist as effective power control tradeoff?

\section{Background literature}

The crucial aspect in evaluating and planning a radio network is the computation of the effect of co-channel interference in the radio links. The amount of interference that can be tolerated determines the required separation distance between co-channel cells and therefore, also the efficiency of the network. Initial analyses of performance was limited to outage probabilities in continuous wave voice communication, taking into account pathloss and flat (nonselective frequency) Rayleigh fading. Within the last two decades, the technique for outage probabilities computation has been sequentially refined $[9,10,11,12]$, considering among other issues, shadowing [13], multiple signal interference $[14,15,16]$ cumulating coherently or incoherently, modulation techniques with error correction 
methods, and more recently, the presence of a dominant line of site propagation path, as it occurs in microcellular networks. Some studies have introduced the stochastic occupation of nearby co-channel cells according to the Erlang traffic laws [17].

Previous studies on cellular system capacity determines outage probability through time consuming Monte Carlo simulation of outage events or by a variety of numerical techniques aimed at generating the signal-to-interference ratio (SIR) distribution $[18,19,20]$. A statistical model for wireless network interference with Poisson spatial distribution of nodes in 1-D, 2-D and 3-D spaces with uniform and non-uniform densities is considered in Mordachev and Loyka [21]. The model which includes parameters such as the average propagation pathloss and the different types of fading, use the power of the nearest (dominant) interferer as a performance measure based on a previous approach proposed in Mordachev [22]. Mordachev [23], instead of relying on the total interference power, observed that when a given number of nearest interferers are completely cancelled, the outage probability exponentially decreases with the number of interferers. This he proved by considering three different models of partial cancellation, with a comparison of their outage probabilities.

In Grant and Cavers [24,25], a detail analytical technique for outage probability computation for the uplink capacity assessment of a generic cellular system with arbitrary size and location of co-channel cells is presented. They assume a flat Rayleigh fading/Log-normal shadowing environment with co-channel interference and implement a simple but accurate geometric approximation in the spatial average. A focus on outage probability computation in GSM-GPRS cellular systems is made in $\mathrm{Ni}$, Liang and Haggman [26], with a presentation of a method that takes into account Rayleigh fading power control (with error) and discontinuous transmission, with or without frequency hopping.

Papandriopoulos, Evans and Dey [27] proposed a new scheme that couples power control with a minimum outage probability multi-user detector. They implement an iterative algorithm that determines the minimum cumulative power of all users with a set of outage probability constraints. Their simulation results show the closeness of the optimal and mapped schemes, the speed of convergence and performance comparisons with other standard receivers. In Song, Cheng and Blostein [28], an exact outage probability analysis of equal gain combination (EGC) in Rayleigh fading with cochannel interference is presented. They provide a more accurate model for interference power calculation. Their model considers pulse-shaped random delays between interference signals, inter-symbol interference, as well as equal and distinct interferer's powers.

A tractable and scalable approximation method for chance constrained stochastic power control problems that arise in power control with outage probability specifications is described in Hsiung, Kim and Boyd [13]. Their method is based on the Chebyshev approximation technique, where they replace the chance (outage probability) constraints with conservative but tractable constraints to form approximate deterministic problems, whose solutions are guaranteed to be feasible to the chance constrained stochastic problems. Their model which handles a variety of shadow fading channel models such as Nikagami/Rician/Weibull/Gamma fading with lognormal/gamma shadowing, strikes a balance between computational complexity and performance, efficiently finding power allocations that meets the outage probability specifications.

The above literature concentrates mainly on analytical solutions to outage probability computation. No evaluation of these computations with respect to a realistic network has been done. This paper therefore studies a realistic cellular network in a macrocellular environment, derives and simulates an outage probability computation model that establishes relationship between specific system parameters, and discusses their effect on the general performance of the system.

\section{Power control}

The link level performance of a user is a function of its signal-to-interference plus noise ratio (SINR). To achieve reliable communication, the SINR or the ratio of the energy per bit to the interference and noise per chip (commonly denoted by $\varepsilon_{b} / I_{o}$ ) should exceed a certain threshold. This threshold depends on the specific code used as well as the multipath channel statistics. For instance, a typical $\varepsilon_{b} / I_{o}$ threshold in the IS-95 system is 6 to $7 \mathrm{~dB}$. In a mobile communication system, the attenuation of both the user of interest and the interferers varies with users' mobility. This is due to varying pathloss and shadow fading effects. To maintain a target SINR, transmit power control is required. The power control problem can be formulated in the network setting as follows: Let $k$ be the total number of users a CDMA system can support. Suppose $k$ is assigned to a cell (base station) $C_{k}$. Let $P_{k}$ denote the transmit power of user $k$ and $g_{k m}$ be the signal attenuation of user $k$ to base station $m$. Then the received energy per chip for user $k$ at all $m$ is simply given by $P_{k} g_{k m} / W$. Using the SINR per chip [29] in Eq.1,

$$
\operatorname{SINR}_{c}=\frac{\varepsilon_{I}^{C}}{\sum_{k>1} \varepsilon_{k}^{c}+N_{o}}
$$

if each user's target $\varepsilon_{b} / I_{o}$ is $\beta$, then the transmit power of the users should be controlled such that

$$
\frac{G P_{k} g_{k}, c_{k}}{\sum_{n \neq k} P_{n} g_{n}, c_{k}+N_{o} W} \geq \beta ; k=1, \ldots . . K
$$

where $G=W / R$, is the processing gain of the system. Moreover, due to constraints on the dynamic range of the transmitting mobiles, there exist a limit on the transmit powers as well

$$
P_{k} \leq \vec{P} ; K=1, \ldots \quad K
$$

These inequalities define the set of all feasible power vectors $\vec{P}=\left(P_{1}, \ldots P_{k}\right)^{t}$. This set is a function of the attenuation of users, and the SINR requirements of the users cannot be simultaneously met if this power set is empty. This condition depicts a system in outage. On the other hand, if this set of feasible powers is non-empty, an optimal solution that will 
conserve energy is that which requires as minimal power as possible.

It can be shown that whenever the feasible set is nonempty, there exist a component wise minimal solution $P^{*}$ in the feasible set, i.e. $P_{k}^{*} \leq P_{k}$, for every user $k$ in any other feasible power vector $\vec{P}$. This fact follows from a basic monotonicity property of the power control problem, which states that when a user lowers it's transmit power, it creates less interference, which benefits all other users in the system.

At an optimal solution $P^{*}$, every user is at the minimal possible power such that their SINR requirements are met with equality. Note that at the optimal point, all the users in the same cell have the same receive power at the BS. It can also be shown that a simple distributed power algorithm will converge to the optimal solution where at each step, each user updates its transmitting power such that its own SINR requirement is just met with the current level of interference. Even if the updates are done asynchronously among the users, convergence is still guaranteed. These results provide theoretical justification to the robustness and stability of the power control algorithms implemented in practice.

Power control and softhandoff [30] minimize the transmit powers required to meet the SINR requirements if there exist a feasible solution for the powers. Otherwise, the system is in outage. The system capacity is the maximum number of users that a system can accommodate for a desired outage probability and a link level $\varepsilon_{b} / I_{o}$ requirement. A system can be in outage due to various random events. For instance, users can be in certain configurations that create a lot of interference on neighboring cells. Also, voice or data users have periods of activity, and too many users can be active in the system at the same time. Another source of randomness emanates from imperfect power control. While it is impossible to have a zero outage probability, it is also desirable to maintain a probability that is slightly below a target threshold. Fortunately, the link level performance of a user in the uplink depends on the aggregate interference at the base station due to many users and the effect of these sources of randomness tends not to be too conservative in admitting users into the network and still guaranteeing a minimal probability of outage. This translates into a larger system capacity.

\section{Data analysis of a realistic cellular network}

Knowledge of observed data is most useful when planning a simulation study. In this section, we analyze data observed from a realistic cellular network - Airtel Nigeria. The essence of working with observed data is to study the outage trend of the cellular system and model important constraint parameter(s) in our design to enhance system efficiency.

For this research, weekly data were gathered for a period of up to 3 months (September-November, 2009), at the various Base Station Controllers (BSCs) for Akwa Ibom State (East region) of Nigeria. Tab.1 shows the average outage occurrences in minutes for the three months. From Tab.1, we observe that signal outages occur in every 105.12 mins (i.e. every $1.75 \mathrm{hrs}$ ) on the average.
Table 1. Average outage duration data measured from the Zain Nigeria network

\begin{tabular}{ccccc}
\hline \multicolumn{1}{c}{ BSC } & Sept & Oct & Nov & \multicolumn{1}{c}{$\begin{array}{c}\text { Avg. } \\
\text { occurrence } \\
\text { time (mins) }\end{array}$} \\
\hline MAKBS09 & 32.80 & 75.34921 & 82.97189 & 63.71 \\
MAKBS11 & 72.40 & 212.6984 & 124.5052 & 136.54 \\
MAKBS12 & 240.17 & 97.65633 & 108.68 & 148.84 \\
MAKBS16 & 117.83 & 136.7182 & 85.24272 & 113.26 \\
MAKBS22 & 24.50 & 91.72245 & 23.09744 & 46.44 \\
MAKBS40 & 168.26 & 94.95422 & 102.5988 & 121.94 \\
\hline $\begin{array}{l}\text { Avg. Time } \\
\text { (mins) }\end{array}$ & 109.33 & 118.18 & 87.85 & 105.12 \\
\hline
\end{tabular}

Tab.2 shows the average outage (\%) for the three months under study. We observe that on the average, there is a 0.0104 likelihood that an outage will occur in the existing system. We also notice that MAKBS12 experienced the highest outage

Table 2. Average $\%$ outage data from the Zain Nigeria network

\begin{tabular}{ccccc}
\hline BSC & Sept & Oct & Nov & $\begin{array}{c}\text { Average \% } \\
\text { outage }\end{array}$ \\
\hline MAKBS09 & 0.33 & 0.75 & 0.82 & 0.63 \\
MAKBS11 & 0.72 & 2.11 & 1.24 & 1.35 \\
MAKBS12 & 2.38 & 0.97 & 1.08 & 1.48 \\
MAKBS16 & 1.17 & 1.36 & 0.85 & 1.12 \\
MAKBS22 & 0.24 & 0.91 & 0.23 & 0.46 \\
MAKBS40 & 1.67 & 0.94 & 1.02 & 1.21 \\
\hline $\begin{array}{l}\text { Average \% } \\
\text { outage }\end{array}$ & 1.08 & 1.17 & 0.87 & 1.04 \\
\hline
\end{tabular}

\section{System Model}

\subsection{Outage probability model}

Let us consider the specific case of averaging users' burstiness. For simplicity, consider a single cell situation with $K$ total number of users, power controlled to a common BS and no out-of-cell inference. It can be seen that the $\varepsilon_{b} / I_{o}$ requirement (i.e., SINR requirement $\beta$ ) of all users is satisfied if

$$
\frac{G Q_{k}}{\sum_{n \neq k} Q_{n}+N_{o} W} \geq \beta \quad k=1, \ldots K
$$

where $Q_{k}=P_{k} g_{k}$ is the received power of user $k$ at the BS, $P_{k}$ is the transmit power of user $k, g_{k}$ is the attenuation of user $k$ 's signal to the base station and $G$ is the processing gain.

Equivalently;

$$
G Q_{k} \geq \beta\left(\sum_{n \neq k} Q_{n}+N_{o} W\right) \quad k=1, \ldots K
$$

Summing over all the inequalities, we obtain the following necessary conditions for $Q_{k}$ : 


$$
[G-\beta(K-1)] \sum_{k=1} Q_{k} \geq K N_{o} W \beta
$$

Therefore, a necessary condition for the existence of feasible powers is $G-\beta(K-1)>O$, or equivalently

$$
K<\frac{G}{\beta}+1
$$

On the other hand, if this condition is satisfied, the powers

$$
Q_{k}=\frac{N_{o} W \beta}{G-\beta(K-1)} ; \quad k=1, \ldots K
$$

will meet the $\varepsilon_{b} / I_{o}$ requirements of all the users. Hence, Eq.7 is a necessary and sufficient condition for the existence of feasible powers to support a given $\varepsilon_{b} / I_{o}$ requirement. Eq.7 yields the interference limited system capacity of a single cell. This implies that, because of the interference between users, there is a limit on the number of users admissible in the cell. Substituting $G=\mathrm{W} / \mathrm{R}$ into Eq.7, we have

$$
\frac{K R}{W}<\frac{1}{\beta}+\frac{1}{G}
$$

The quantity $\frac{K R}{W}$ is the overall spectral efficiency of the system (in bits/s/Hz). Since the processing gain $\mathrm{G}$ of a CDMA system is typically large, Eq.9 shows that the maximal spectral efficiency is approximately $1 / \beta$. Thus, a typical $\varepsilon_{b} / I_{o}$ requirement $\beta$ of $6 \mathrm{~dB}$ translates into a maximum spectral efficiency of $0.25 \mathrm{bits} / \mathrm{s} / \mathrm{Hz}$.

Let us now illustrate the effect of user burstiness on the system capacity and the spectral efficiency in a cell setting. Recall that we have assumed that all $K$ users are active at all times. Now, suppose each user is active and has data to send only with probability $p$ with mutually exclusive activities and independent voice users, for instance, if users are typically talking $3 / 8$ th of the time and if the voice coder can detect silence, there is no need transmitting data during the quiet periods. If we let $V_{k}$ be the indicator random variable for user $k$ 's activity, and $V_{k}=1$, when user $k$ is transmitting or $V_{k}=0$, otherwise, using Eq.7, the $\varepsilon_{b} / I_{o}$ requirements of the users can be met only when

$$
\sum_{k=1}^{K} V_{k}<\frac{G}{\beta}+1
$$

Whenever this constraint is not satisfied, the system is in outage. If the system wants to guarantee that an outage would occur, then the maximum number of users admissible in the network is $\frac{G}{\beta}+1$. This case is similar to when users are active at all times. However, more users can be accommodated if a minimal outage probability $P_{\text {out }}$ can be tolerated: this number $K^{*}\left(P_{\text {out }}\right)$ is the largest $K$ such that

$\operatorname{Pr}\left[\sum_{k=1}^{K} V_{k}>\frac{G}{\beta}+1\right] \leq P_{\text {out }}$

The random variable $\sum_{k=1}^{K} V_{k}$ is binomially distributed with mean $K p$ and standard deviation $\sqrt{K p(1-p)}$, where $p(1-p)$ is the variance of $V_{k}$. When $P_{\text {out }}=0, K^{*}\left(P_{\text {out }}\right)=\frac{G}{\beta}+1$. If $P_{\text {out }}>0$, then a larger value of $K^{*}\left(P_{\text {out }}\right)$ can be chosen. It is straight forward to calculate $K^{*}\left(P_{\text {out }}\right)$ numerically for a given $P_{\text {out }}$. It is also interesting to see what happens to the spectral efficiency when the bandwidth of the system $W$ scales with the rate $R$, with each user fixed. In this regime, there are many users in the system and it is reasonable to apply a Gaussian approximation to $\sum_{k=1}^{K} V_{k}$. Hence,

$\operatorname{Pr}\left[\sum_{k=1}^{K} V_{k}>\frac{G}{\beta}+1\right] \approx Q\left[\frac{\frac{G}{\beta}+1-K p}{\sqrt{K p(1-p)}}\right]$

The overall spectral efficiency of the system is given by

$\rho=\frac{K p R}{W}$

since the mean rate of each user is $p^{R}$ bits/s from using the approximation in Eq.12. From Eq.11, we can solve for the constraint on the spectral efficiency $\rho$ :

$\rho \leq \frac{1}{\beta}\left[1+Q(x)^{-1}\left(P_{\text {out }}\right) \sqrt{\frac{1-P}{p K}}-\frac{1}{K p}\right]^{-1}$

As seen in Eq.9, the value $1 / \beta$ is the maximum spectral efficiency if each user is non-bursty and transmitting at a constant rate equal to the mean rate $p^{R}$ of the bursty user. However, the actual spectral efficiency in the system with burstiness is different from this, by a factor of

$\left(1+Q(x)^{-1}\left(P_{\text {out }}\right) \sqrt{\frac{1-p}{p K}}-\frac{1}{K p}\right)^{-1}$ 
This loss in spectral efficiency is due to a need to admit fewer users to cater for the burstiness of the traffic. This "safety margin" is larger when the outage probability requirement $P_{\text {out }}$ is more stringent. More importantly, for a given outage probability, the spectral efficiency approaches $1 / \beta$ as the bandwidth $W$ (and hence the number of users $K$ ) scales. When there are many users in the system, interference averaging occurs: the fluctuation of the aggregate interference is smaller relative to the mean interference level. Since the link level performance of the system depends on the aggregate interference, less excess resources need to be set aside to accommodate the fluctuations. This is a manifestation of the familiar principle of statistical multiplexing.

\subsection{Spatial traffic and basic relations}

To estimate the coverage of CDMA cells in a network planning context, we consider the customer population on a two dimensional surface, to constitute a spatial homogeneous Poisson process. Thus, the distribution of the random variable $K_{Z}$ of calls on a surface with area $Z$ is Poisson distributed as:

$P\left(K_{Z}=k\right)=\frac{(\lambda Z)^{k}}{k !} e^{-\lambda Z}$

where $\lambda$ (in calls per $\mathrm{Km}^{2}$ ) denotes the spatial traffic intensity. The distribution of $K_{Z}$ given in Eq.16 is valid at any arbitrary observation instant. Based on this Poisson process assumption, we now consider a cell modeled by a circle with radius $R_{C}$. An active call is assumed to be on the circle with k-1 connections. The corresponding coverage area is $A_{C}=\pi R_{C}^{2}$, where $A_{C}$ and $R_{C}$ are random variables. To precisely describe this process mathematically, we define the random variable $A$ as the surface of the smallest circle containing $k$ points. Due to the property of the spatial Poisson process, the size of the surface $A$ is distributed as an Erlang-distribution of order $k$ :

$$
A(y)=P(A \leq y)=1-\sum_{i=0}^{k-1} \frac{(\lambda y)^{i}}{i !} \cdot e^{-\lambda y}
$$

with probability density function

$$
a(y)=\frac{d}{d y} A(y)=\frac{\lambda(\lambda y)^{k-1}}{(k-1) !} \cdot e^{-\lambda y}
$$

It is more useful, however, to consider the radius of the cell rather than its surface, as this directly translates to the distance between the customer and base station. The distribution of the radius $R_{C}$ can be derived as

$$
R_{C}(x)=P\left(R_{C} \leq x\right)=P\left(A \leq \pi x^{2}\right)=1-\sum_{i=0}^{k-1} \frac{\left(\lambda \pi x^{2}\right)^{i}}{i !} \cdot e^{-\lambda \pi x^{2}}
$$

with probability density function
$r_{C}(x)=\frac{d}{d x} R_{C}(x)=\frac{d}{d y} \frac{d y}{d x} R_{C}(x)=\frac{\lambda\left(\lambda \pi x^{2}\right)^{k-1}}{(k-1) !} \cdot e^{-\lambda \pi x^{2}}(2 \pi x)$

From Eq.20, we can now compute the probability that there exist a cell radius $x$, for a cell currently supporting $k$ calls, assuming an intensity $\lambda$.

\subsection{CDMA cell coverage}

Considering the two-dimensional customer traffic process discussed above, it is possible to establish an estimate of the coverage area of a cell in a CDMA network. The outage probability

$P_{\text {out }}(x, k)=Q(x)\left(\frac{\left(\frac{G}{\beta}+1-K p\right)}{\sqrt{K p(1-p)}}\right)$

where Q represents the Q-function is given by Ekpenyong, Umoren and Isabona [31]:

$Q(x)=\frac{1}{\sqrt{2 \pi}} \int_{x}^{\infty} e^{-\frac{t^{2}}{2}} d t$

and will be used as the criterion to define the boundary of the cell. Considering the outage perceived by a customer at distance $x$ from the BTS, we can formulate $P_{\text {out }}(x)$ as:

$$
p_{\text {out }}(x)=\sum_{k} P_{\text {out }}(x, k) \cdot P(" k \text { calls in cell with radius } x ")
$$

The derivation of the probability of having $k$ customers in a cell based on certain traffic behaviour is obtained from a spatial Poisson process earlier described. Similarly we describe $P_{\text {out }}(k)$ as

$$
P_{\text {out }}(k)=\int_{o}^{\infty} P_{\text {out }}(x, k) \cdot P\left(\text { "radius of cell with } k \text { calls is } x^{\prime \prime}\right) d x
$$

With the Poisson process, we now have a mechanism to describe the probability of having $k$ calls in the cell with radius $x$, and the probability that the cell with $k$ calls is $x$. First, we look at a cell with radius $R_{C}=x$. The probability of having $k$ connections in the cell with radius $x$ is simply Poisson distributed, as shown in Eq.16. The overall unconditioned outage probability for this cell can then be derived as

$$
P_{\text {out }}(x)=\sum_{k=1}^{\infty} P_{\text {out }}(x, k) \cdot \frac{\left(\lambda \pi x^{2}\right)^{k}}{k !} \cdot e^{-\lambda \pi x^{2}}
$$

The resulting outage probability in Eq.24 no longer depend on the number of calls in the cell as in Eq.21, but only depends on the distance from the base station and the intensity of the cluster process. This translates to an assumed traffic value for the area of the cell. Therefore, it is enough to know the environment of the cell, such as urban or suburban, and map this value to a certain value of $\lambda$. 
We now focus on the coverage area for the CDMA cell for a given number of $k$ active calls. From a network design viewpoint, the coverage corresponds to a chosen outage probability, which can be derived by combining Eq.20 and Eq.21, i.e.:

$$
P_{\text {out }}(k)=\int_{o}^{\infty} P_{\text {out }}(x, k) \cdot r_{C}(x) d x
$$

Eq.25 establishes a relationship between probability of outage and number of calls. Here, it is no longer necessary to know the distances of the individual customers as these are being implicitly represented by the Poisson process.

\section{Model simulation and results interpretation}

\subsection{Data instrument, field survey and techniques}

(i) Base map and field survey of base stations location using GPS: The base map of Akwa Ibom state is used in this research. The map, showing the local government areas (LGAs), was obtained from the Akwa Ibom State Ministry of Lands and Environment's Geographic Department. The field techniques involve a survey of Airtel's base stations (masts) location in Akwa Ibom State and taking the required measurements (longitude and latitude) using the GPS device.

(ii) Map processing: The raw map was processed in the following order: scanning, geo-referencing and digitization. The Digital Elevation Model (DEM) of the study area is used to determine the slope pattern of Akwa Ibom State. This elevation model is useful for the appropriate identification of mast locations and slope pattern analysis.

\subsection{Simulation input parameters}

The proposed model was simulated using observed data from the Airtel Nigeria network. Tab. 3 shows the input parameters and their respective values. Other empirical parameters measured from the environment under study are also presented.

Table 3. Simulation input parameters

\begin{tabular}{lc}
\hline \multicolumn{1}{c}{ Parameter } & Value \\
\hline Processing gain $(\mathrm{G})$ & $23 \mathrm{~dB}$ \\
Number of active callers $(\mathrm{K})$ & $10-60$ \\
SIR requirement $(\beta)$ & $5 \mathrm{~dB}$ \\
Outage probability average from Zain $(\mathrm{t})$ & 0.0104 \\
Transmit power $(\mathrm{P})$ & $3 / 8 \mathrm{~dB}$ \\
Traffic intensity $(\lambda)$ & $(10,20,30) \mathrm{Erlang} / \mathrm{s}$ \\
Cell radius $(\mathrm{x})$ & $(0.1-0.6) \mathrm{km}$ \\
\hline
\end{tabular}

\subsection{Performance analysis}

In Tab.4 and Tab.5, we summarize the outage probabilities of the observed data from the Airtel network and the model simulation results. We discover that the existing cellular system could be enhanced further, as simulation results show $81.02 \%$ reduction in signal outage. We also predict with a 95\% confidence interval that subsequent observations and simulations are most likely to fall within these limits.
Table 4. Observed outage performance data

\begin{tabular}{lrrr}
\hline BSC & $\begin{array}{l}\text { No. of } \\
\text { BS }\end{array}$ & \multicolumn{1}{l}{$\begin{array}{l}\text { Percentage } \\
\text { outage }\end{array}$} & Confidence interval (CI) \\
\hline MAKBS09 & 22 & 0.822559 & $0.822559 \pm 0.493992$ \\
MAKBS11 & 26 & 1.195425 & $1.195425 \pm 0.4695$ \\
MAKBS12 & 25 & 1.391686 & $1.391686 \pm 0.609551$ \\
MAKBS16 & 27 & 1.494473 & $1.494473 \pm 0.697615$ \\
MAKBS22 & 24 & 1.168275 & $1.168275 \pm 0.579641$ \\
MAKBS40 & 24 & 1.166062 & $1.166062 \pm 0.441852$ \\
\hline & Average & 1.206413 & \\
\hline
\end{tabular}

Table 5. Simulated outage performance data

\begin{tabular}{lccc}
\hline BSC & \multicolumn{1}{l}{$\begin{array}{l}\text { No. of } \\
\text { BS }\end{array}$} & \multicolumn{1}{l}{$\begin{array}{l}\text { Percentage } \\
\text { outage }\end{array}$} & Confidence interval (CI) \\
\hline MAKBS09 & 22 & 0.155626 & $0.155626 \pm 0.09346$ \\
MAKBS11 & 26 & 0.240349 & $0.240349 \pm 0.08883$ \\
MAKBS12 & 25 & 0.263303 & $0.263303 \pm 0.11533$ \\
MAKBS16 & 27 & 0.279665 & $0.279665 \pm 0.13199$ \\
MAKBS22 & 24 & 0.214004 & $0.214004 \pm 0.10967$ \\
MAKBS40 & 24 & 0.220616 & $0.220616 \pm 0.0836$ \\
\hline \multicolumn{4}{c}{ Average } \\
\hline
\end{tabular}

GIS maps showing the distribution of the signal outage rates at various base stations in Akwa Ibom State for both observed and simulated data are presented in Fig.1 and Fig.2 respectively. A qualitative analysis reveals that, users experience frequent signal outages especially in urban and poor terrain locations (see Fig.1). This could be attributed to lack of effective power control models, increase in mobile users, populated buildings/structures and equipment quality. Fig.2 when compared with Fig.1 shows a remarkable improvement on the existing system's performance as signal outages are effective minimized. The software tool used to provide this solution include the ArcGIS and Digital Elevation Model (DEM) software.

In Fig.3, outage probability is plotted against number of active users, taking data rates as parameter of distinction between data traffic and voice traffic respectively. The result shows the robustness of our proposed computation. A plot of spectral efficiency with respect to the number of callers is shown in Fig.4. We observe from this graph that the spectral efficiency of the network reduces as more subscribers use the network. The decrease in spectral efficiency can be well traced to insufficient network resources to accommodate more active number of users.

In Fig.5, we explore the impact of traffic intensity on the network as the cell radius is varied. As expected, the QoS decreases as the traffic intensity increases. Furthermore, the choice of outage probability will depend on the area over which the coverage area of the cell is desired. In this analysis, power outage is maintained along the cell edge, which shows the extent to which the outage can be maintained from the BS to the edge of the cell. 


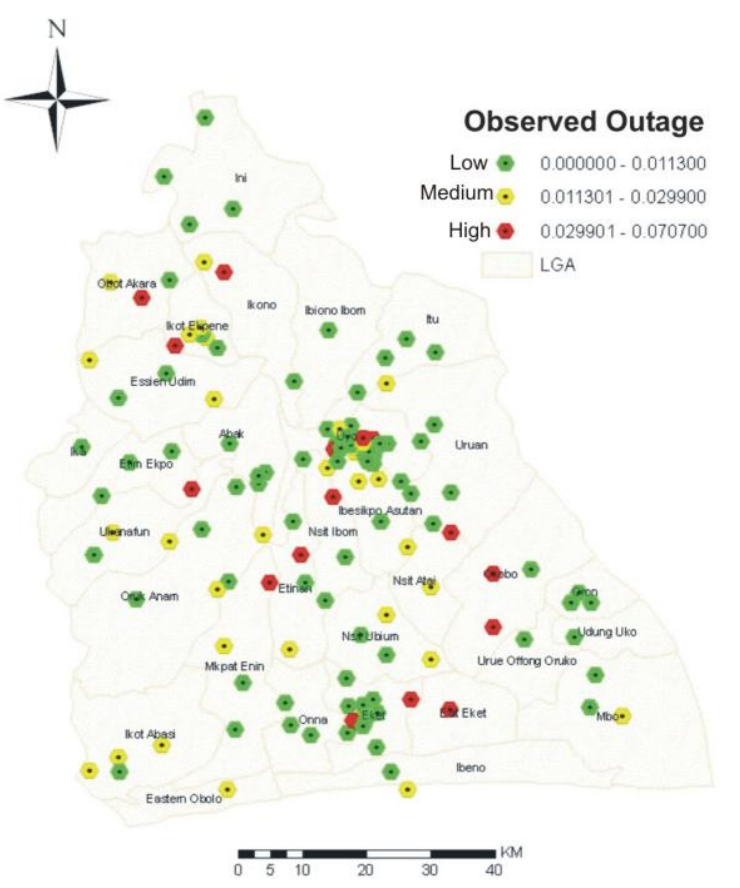

Fig. 1. A GIS map showing the distribution of observed signal outage rates for BSs in Akwa Ibom State of Nigeria

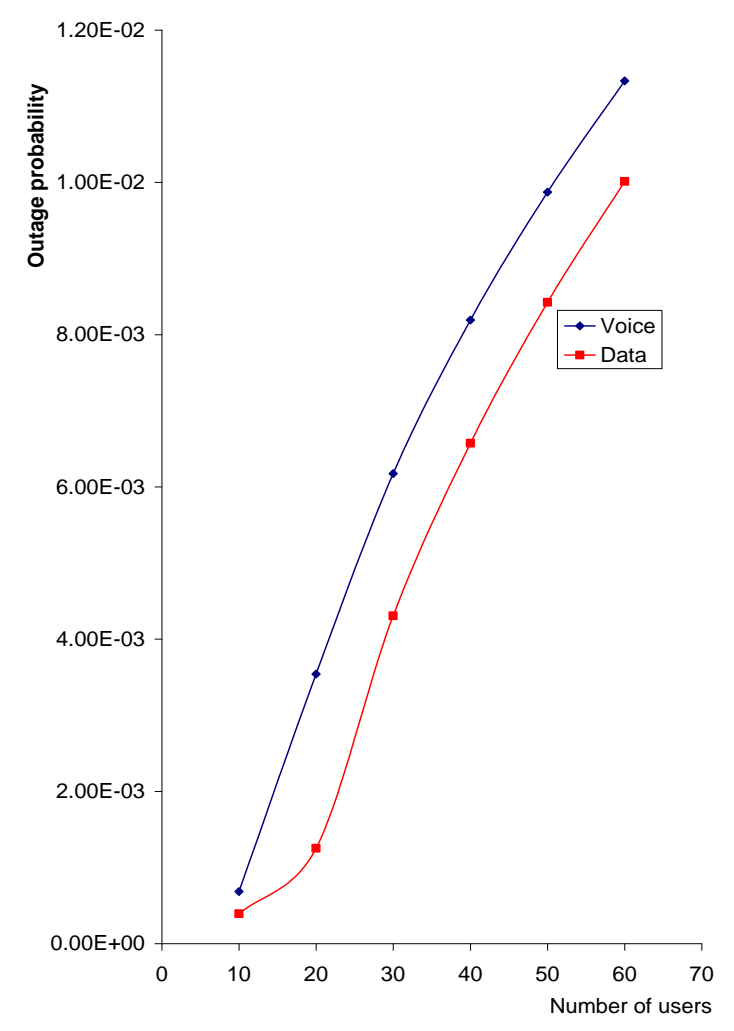

Fig. 3. A graph of outage probability vs. number of users
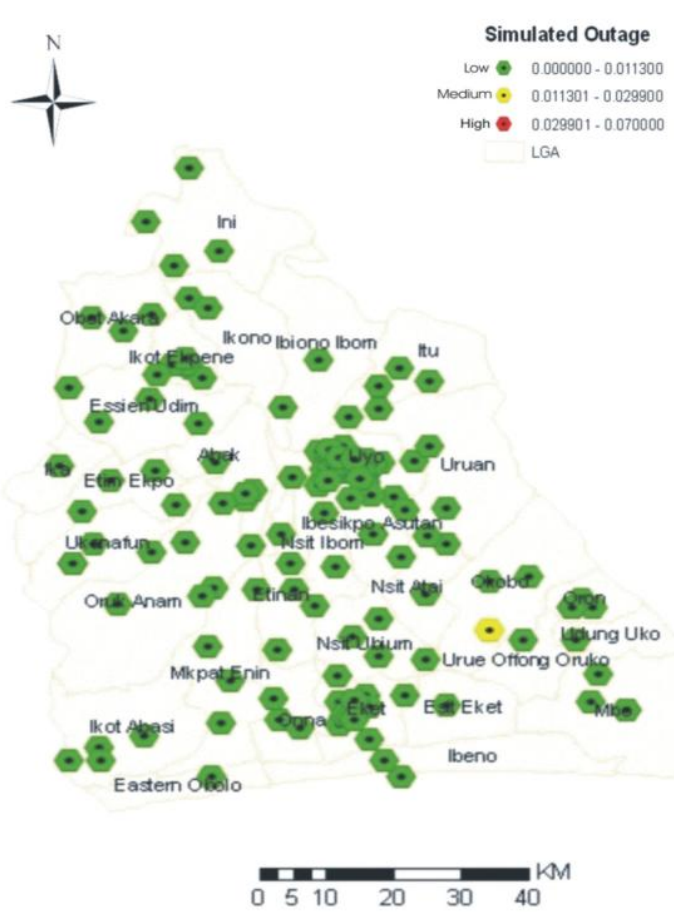

Fig. 2. A GIS map showing the distribution of simulated signal outage rates for BSs in Akwa Ibom State of Nigeria

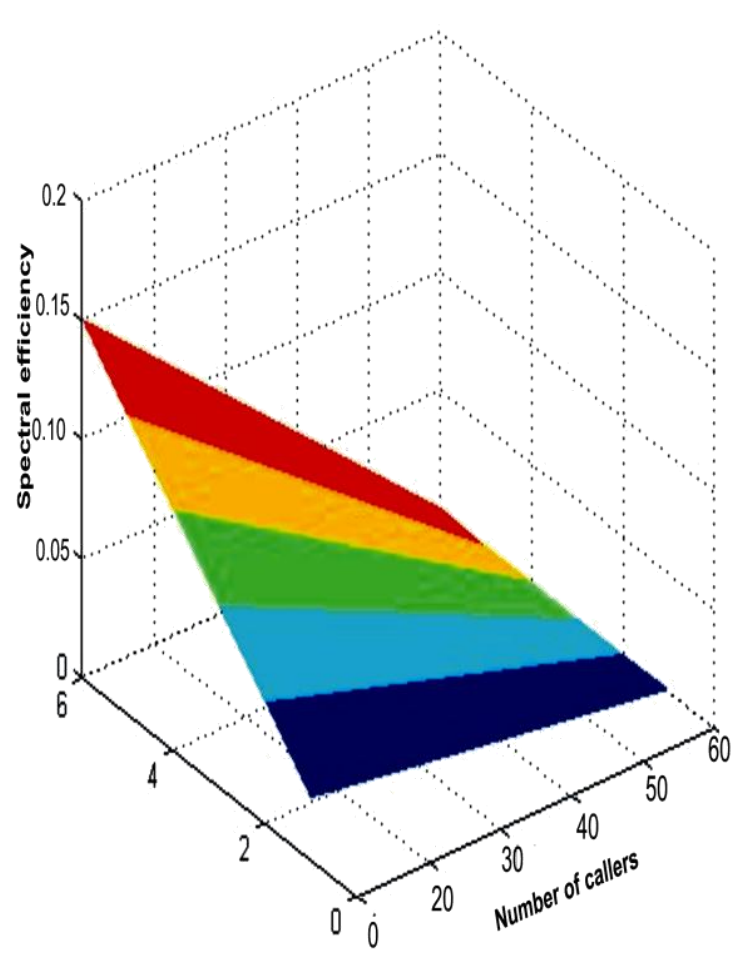

Fig. 4. A graph of spectral efficiency vs. number of callers 


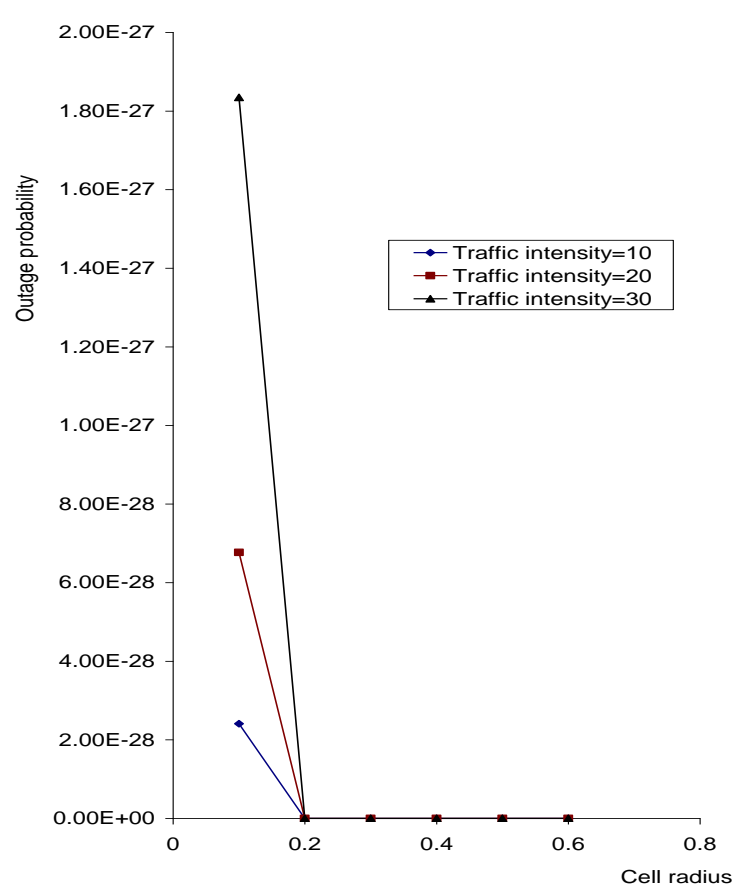

Fig. 5. A graph of outage probability vs. cell radius

\section{Conclusion}

The CDMA wireless technology satisfies the increasing demands of today's voice and data transfer services with excellent roaming and improved voice quality at a much higher bandwidth. However, despite all of these, its infrastructure is still not free from performance flaws arising from its ever increasing users, which results in co-channel interference and leads to system outages. We have presented a modeling approach for evaluating outage probability in the presence of co-channel interference in a realistic macrocellular environment and implemented a GIS solution of this approach for base stations in Akwa Ibom State (East region) of Nigeria using the Airtel network as a case study. We have also provided an accurate methodology that can efficiently be applied to other wireless applications with arbitrary characteristics for parametric modeling in interference limited environments.

\section{Acknowledgment}

We are grateful to staff of Airtel Nigeria, for their assistance during the data gathering (field work) phase of this research.

\section{References}

1. Gupta, P. and Kumar, P. R. (2000). The Capacity of Wireless Networks. IEEE transaction on Information Theory. 46 (2): $388-404$

2. Sousa, E. S., Silvester, J. A. (1990). Optimum Transmission Ranges in a Direct-Sequences Spread-Spectrum Multitop Packet Radio Network. IEEE JSAC. 8(5): 762-771.

3. Sousa, E. S. (1992). Performance of A Spread Spectrum packet radio Network Link in a Poisson Feild of Interferers. IEEE Transaction on Information Theory. 38(6): $1743-1754$.

4. Ilow, J., Hatzinakos, D. (1998). Analytical Alpha-Stable Noise Modeling in a Poisson Field of Interferers or Scatterers. IEEE Transaction on Signal Processing. 46(6): 1601-1611.

5. Ilow, J.; Hatzinakos, D.; and Venetsanapoulas (1998). Performance of FH SS Radio Networks with Interference Modeled as a mixture of Gaussian and Alpha - Stable Noise. IEEE Transaction on Communications. 46(4): $509-520$.

6. Weber, S. P.; Yang, X; Andrews J. G.; de Veciana, G. (2005). Transmission Capacity of Wireless Ad Hoc Networks with Outage Constraints. IEEE Transaction on Information Theory. 51 (12):40914102.

7. Weber, S. P., Andrews J. G.; Yang X. and de Veciana, G. (2007) Transmission Capacity of Wireless Ad Hoc Networks with Successive Interference Cancellation. IEEE Transaction on Information Theory. 53(8): 2799-2814.

8. Zhang, J. and Host-Madsen, A. (2005). Capacity Bounds and Power Allocation for Wireless Relay Channels. IEEE Transactions on Information Theory. 51(6): 2020-2040.

9. Linnartz, J-P. M. G. (1993). Narrowband Land mobile Radio Networks. Artech, House, Norwood, MA..

10. Prasad, R. and Kegel, A. (1993). Effects of Rician Faded and LogNormal Shadowed Signals on Spectrum Efficiency in Micro-Cellular Radio. IEEE Transactions on Vehicular Technology. 42(3): 274-285.

11. Ho, M. J. and Stuber, G. L. (1993). Co-Channel Interference of Microcellular System on Shadowed Nakagami Fading Channels. In Proceedings of $43^{\text {rd }}$ IEEE Conference on Vehicular Technology: 568571

12. Chennakeshu, S., Hassan, A. and Anderson, J. (1993). Capacity Analysis of A Mixed Mode Slow Frequency Hopped Cellular System. In Proceedings of IEEE Conference on Vehicular Technology: 540543.

13. Hsiung, K-L., Kim, S. J. and Boyd, S. (2008). Power Allocation with Outage Probability Specifications in Wireless Shadowed Fading Channels via Geometric Programming. Research Report, Information Systems Laboratory, Stanford University.
14. Abu-Dayya, A. A. and Beaulieu, N. C. (1992). Outage Probabilities of Diversity Cellular Systems with Co-channel Interference in Nakagami Fading. IEEE Transactions on Vehicular Technology. 41 (4): 343-355.

15. Daikoku, K. and Ohdate, O. (1983). Optimal Channel Reuse in Cellular and Land Mobile Radio Systems. IEEE Transactions on Vehicular Technology. 32 (3): 217-224.

16. Prasad, R., Kegel, A. and Arnbak, J. C. (1991). Improved Assessment of Interference Limits in Cellular Radio Performance. IEEE Transactions on Vehicular Technology. 40(2): 412-419.

17. Nathan, B., Ran, G. and Freedman, A. (2002). Unified Approach of GOS Optimisaation for Fixed Wireless Access. Vehicular Technology, IEEE Transactions. 51(1): 200-208.

18. Yeh, Y-S. and Schwartz, S. C. (1984). Outage Probability in Mobile Telephony Due to Multiple Lognormal Interferers. IEEE Transactions on Communication. 32 (4): 380-388.

19. Gilhousen, K. S., Viterbi, A. J., Jacobs I. M., Padovani, R. (1991). On the Capacity of A Cellular CDMA System. IEEE Transactions on Vehicular Technology. 40 (2): 303-312.

20. Chennakeshu, S.; Hassan, A. A.; Anderson, J. B. and Gudmundson, B. (1996). Capacity Analysis of a TDMA-based Slow FrequencyHopped Cellular System. IEEE Transactions on Vehicular Technology. 45(3): 531-542.

21. Mordachev, V. and Loyka, S. (2009). On node density Outage Probability Tradeoff. IEEE Journal on Selected Areas in Communication. 27 (7): 1120-1131.

22. Mordachev, V. I (1990). Typical models of Electromagnetic Environments for Spatially Scattered Radio Transmitters. In Proceedings of the $10^{\text {th }}$ Worclaw Symposium on EMC: 409-414.

23. Mordachev, V. (2000) Mathematical models for Radio signals Dynamic Range Prediction in Space-Scattered Mobile Radiocommunication Networks. IEEE Vehicular Technology Conference Fall, Boston 2: 904-911.

24. Grant, S. J. and Cavers, J. K. (1999). Increased Uplink Capacity for TDMA System Through Joint Detection and Diversity Arrays. In Proceedings of IEEE Vehicular Technology Conference, Armsterdan, The Netherlands. 1: 623-627.

25. Grant, S. J. and Cavers J. K. (2000). Further Analytical Results on the Joint Detection of Co-channel Signals using Diversity Arrays. IEEE Transactions on Communications. 48 (11): 1788-1792.

26. Ni, S., Liang, Y. and Haggman, S-G. (2000). Outage Probability in GSM-GPRS Cellular Systems With and Without Frequency Hopping. 
International Journal on Wireless Personal Communications. 14 (3): 215-234.

27. Papandriopoulos, J.; Evans, J. and Dey, S. (2003). Iterative Power Control and Multiuser Detection with Outage Probability Constraints. In Proceedings of IEEE International Conference on Communications. 4: 2509-2513.

28. Song, Y., Cheng, J. and Blostein S-D. (2003). Exact Outage Probability for Equal Gain Combining with Co-channel Interference in Rayleigh Fading. IEEE Transactions on Wireless Communications. 2 (5): $865-870$.

29. Viterbi, A. J. (1995). CDMA: Principles of Spread Spectrum Communication. Adison-Wesley Inc.

30. van Cauwenberge, S. N. P. (2003). Study of Soft Handover in UMTS. Masters Thesis, Danmarks Tekniske Universitet.

31. Ekpenyong, M., Umoren, E. and Isabona, J. (2009). A Rain Attenuation Model for Predicting Fading Effect on Wireless Communication Systems in the Tropics. Nigerian Journal of Space Research. 6: 21-32. 